\title{
Role of ofatumumab in treatment of chronic lymphocytic leukemia
}

This article was published in the following Dove Press journal:

Journal of Blood Medicine

29 April 2011

Number of times this article has been viewed

\author{
Marays Veliz \\ Javier Pinilla-Ibarz \\ H Lee Moffitt Cancer Center \\ and Research Institute, Tampa, \\ FL, USA
}

Correspondence: Javier Pinilla-lbarz

$\mathrm{H}$ Lee Moffitt Cancer Center

and Research Institute, 12902

Magnolia Drive, Tampa, FL 33612, USA

$\mathrm{Tel}+$ I 8I3-745-3880

Email javier.pinilla@moffitt.org

\begin{abstract}
The management of chronic lymphocytic leukemia (CLL) has dramatically improved in the past decade with the addition of anti-CD20 monoclonal antibodies to the treatment armamentarium. Ofatumumab is a novel anti-CD20 monoclonal antibody recently approved in the US and Europe for the treatment of CLL refractory to alemtuzumab and fludarabine. Preclinical data showed improved complement-dependent cytotoxicity and antibody-dependent cellular cytotoxicity compared with rituximab. Clinical studies have shown single-agent activity for ofatumumab in CLL and in other low-grade non-Hodgkin's lymphomas. Combination studies are being conducted to enhance the therapeutic efficacy of ofatumumab. This paper reviews some of the key clinical studies that led to approval of ofatumumab, and future directions.

Keywords: ofatumumab, chronic lymphocytic leukemia, efficacy, safety
\end{abstract}

\section{Introduction}

Chronic lymphocytic leukemia/small lymphocytic lymphoma (CLL/SLL) is the most common leukemia in the Western hemisphere, with an age-adjusted incidence rate of 4.2 per 100,000 men and women per year. ${ }^{1}$ It is a monoclonal B cell malignancy that affects mainly older individuals, with a median age at diagnosis of 72 years. The spectrum of this disease varies from a simple lymphocytosis to splenomegaly and/or cytopenias. Some patients have an indolent course, while others have a more accelerated course. In either case, the disease relapses frequently, and there is considerable morbidity from the disease and the treatments themselves. ${ }^{2}$

Several predictive factors have been identified in CLL. In addition to clinical staging, traditional prognostic factors for identifying high risk of disease progression have included elevated serum levels of beta-2 microglobulin, soluble CD23, diffuse bone marrow infiltration, short lymphocyte doubling time, and high levels of zeta-associated protein on the surface of malignant cells. ${ }^{3,4}$ More recently, the presence of certain cytogenetic abnormalities identified by fluorescence in situ hybridization analysis and mutational status of immunoglobulin heavy chain have become a more meaningful predictor of disease progression and duration of response to therapy. ${ }^{5}$

The median survival is highly variable, with some patients following an indolent course with a survival of over 20 years, whereas others exhibit an aggressive behavior with survival less than three years. The management of patients with CLL/SLL is currently undergoing profound changes. Historically, the approach to management of CLL focused on reducing tumor bulk and controlling symptoms, while maintaining a good quality of life. Treatment options for decades included alkylating 
agents, purine analogs, or a combination of the two. More effective combination regimens, such as fludarabine, cyclophosphamide, and rituximab, have recently been shown to prolong progression-free survival and overall survival, thereby changing the treatment paradigm to one with a goal of complete elimination of the disease in patients who are younger or older but physically fit. Because there is no proven survival benefit of early treatment of asymptomatic patients, most patients are followed by watchful waiting until symptoms develop. Although most patients with CLL respond to first-line therapy, all patients eventually relapse, after which the therapeutic options are limited and the prognosis is particularly poor after development of fludarabine-refractory disease.

\section{Preclinical data}

Addition of the CD20 monoclonal antibody, rituximab, to chemotherapy for CLL has improved outcomes, particularly in early lines of therapy. Typically CLL cells have low CD20 expression, which helps explain why the efficacy of rituximab monotherapy in CLL is limited, potentially in part because of reduced cell lysis via complement-dependent cytotoxicity, which is dependent on CD20 expression.

Ofatumumab is an $\operatorname{IgG} 1 \kappa$, fully humanized CD20 monoclonal antibody that targets an epitope distinct from the epitope recognized by rituximab. The antibody is generated via transgenic mouse and hybridoma technology and produced in a recombinant murine cell line (NS0) using standard mammalian cell cultivation and purification technologies. ${ }^{6}$ The mechanism of action of ofatumumab was studied indepth, and compared with that of the marketed chimeric anti-CD20 monoclonal antibody, rituximab. Ofatumumab binds specifically to epitopes which encompass the amino acid residues 163 and 166 in the second extracellular loop of the CD20 molecule. Ofatumumab induces crosslinking of CD20 molecules and relocation of these CD20 molecules to the so-called lipid rafts. ${ }^{7}$ The translocation of CD20 into lipid rafts is considered important for induction of cell signaling and more effective complement activation. ${ }^{8}$ Differences in antibody function between various anti-CD20 antibodies might be explained by their distinct ability to induce relocation of the CD20 molecules within the lipid rafts. The binding of ofatumumab induces cell death, primarily through complement-dependent cytotoxicity and antibody-dependent cellular cytotoxicity and not by apoptosis. Ofatumumab has similar antibody-dependent cellular cytotoxicity when compared with rituximab, but delivers stronger complement-dependent cytotoxicity in in vitro models, even in malignant B cells with low CD20 expression levels. ${ }^{6,9}$ Laboratory studies have shown ofatumumab to be effective at inducing lysis of several B cell lines, as well as being able to kill fresh CLL B cells resistant to rituximab. . $^{6,9,10}$

Further studies have demonstrated that although ofatumumab and rituximab bind the same antigen, ofatumumab dissociates from its target at a slower rate compared with rituximab, ${ }^{6}$ and binds a novel, membrane-proximal epitope. In experiments using radiolabeled antibodies, more than $70 \%$ of ofatumumab, but only $30 \%$ of rituximab, remained bound to deoxyhypusine hydroxylase cells after three hours. Epitope mapping has indicated that ofatumumab binds an epitope located closer to the N-terminus of CD20 compared with the location targeted by rituximab, and includes an extracellular loop of the antigen. It is hypothesized that the complement-dependent potent cytotoxicity of ofatumumab may be due to both the slow off-rate and the precise location of binding on CD20 compared with rituximab. ${ }^{10}$

To evaluate the in vivo efficacy of ofatumumab, its effect was tested in a mouse xenograft model. ${ }^{11}$ Severe combined immunodeficiency mice were first injected with Daudi B cells transfected with luciferase, and then treated with either $0.5 \mathrm{mg} / \mathrm{kg}$ of ofatumumab or anti- keyhole limpet hemocyanin monoclonal antibody as a control. Tumor growth was assessed by bioluminescence. A single dose, resulting in an initial plasma antibody concentration of $5 \mu \mathrm{g} / \mathrm{mL}$, expected to result in full target saturation, effectively inhibited human B cell tumor development. Tumor growth resumed when plasma concentrations dropped below levels that are expected to result in half-maximal saturation. Compared with the control group, the ofatumumab cohort showed delayed tumor induction (3-4 weeks) and lower tumor growth rate. In cynomolgus monkeys, initial depletion of circulating and tissue-residing $\mathrm{B}$ cells required a relatively high dose level. A $1.25 \mathrm{mg} / \mathrm{kg}$ intravenous (IV) dose administered daily for four days effectively depleted B cells. Initial plasma concentrations were approximately $50 \mu \mathrm{g} / \mathrm{mL}$, and repopulation of $\mathrm{B}$ cell compartments only became detectable when ofatumumab levels dropped below $10 \mu \mathrm{g} / \mathrm{mL}$. The cell count did not return to a normal level until 96 days later, but the animals developed primate antihuman antibodies. The results of these animal studies suggested that high initial dosing would be necessary to saturate CD20, but maintenance of a plasma concentration at $5-10 \mu \mathrm{g} / \mathrm{mL}$ maintains target saturation on circulating cells and is probably sufficient for sustained biological activity. ${ }^{11}$ These observations may provide a rationale for establishing dosing schedules for maintenance 
immunotherapy following initial depletion of CD20-positive tumor cells. These data suggest that ofatumumab may deplete B cells for a longer period of time than rituximab, which may lead to a longer duration of treatment response and could potentially result in a smaller dose being needed. Ofatumumab is a human monoclonal antibody with very low anticipated immunogenicity. Therefore, subjects are not expected to produce human antihuman antibodies to the same degree as the human antichimeric antibodies seen in some subjects following treatment with rituximab. Based on these in vivo studies, clinical studies of ofatumumab were initiated in different diseases, including B cell malignancies, such as CLL. The following section reviews the current clinical data on ofatumumab in CLL, as well as future therapeutic directions.

\section{Clinical studies of ofatumumab in CLL}

The Table 1 summarizes completed and ongoing clinical studies of ofatumumab in CLL/SLL. A Phase I/II doseescalating study evaluating ofatumumab in patients with relapsed and refractory CLL was initiated at 12 sites in the US and Europe. This study evaluated 33 patients in three different dosing cohorts. ${ }^{12}$ The majority of patients (27/33) received a total of four doses administered once weekly. The response rate for the cohort receiving an initial dose of $500 \mathrm{mg}$ followed by three $2000 \mathrm{mg}$ doses was $50 \%$, with partial remission observed in 13/26 patients. The drug was well tolerated, with the majority of adverse events being related to infusion reactions and Grade 1 and 2 infections.

A second larger study was done evaluating patients with CLL refractory to alemtuzumab and fludarabine. The interim analysis of this pivotal international study evaluated a total of 138 patients ( 59 were refractory to fludarabine and alemtuzumab [FA-ref] and 79 were refractory to fludarabine but did not receive treatment with alemtuzumab due to bulky disease [BF-ref]). ${ }^{13}$ Ofatumumab was administered as eight weekly infusions followed by four monthly infusions. The first infusion was given as a $300 \mathrm{mg}$ dose and the subsequent infusions were given at a dose of $2000 \mathrm{mg}$. The objective response rate was found to be $58 \%$ and $47 \%$ in the FA-ref and BF-ref groups, respectively. All of these were partial responses, with the exception of one complete response. Complete resolution of constitutional symptoms and improved performance status occurred in 57\% and $48 \%$ of FA-ref and BF-ref patients, respectively. The response was also significant in those who received prior rituximab therapy (objective response rate 54\% in the FA-ref patients and $44 \%$ in the BF-ref patients) as well as those who received prior treatment with the combination of fludarabine, cyclophosphamide, and rituximab (objective response rate of $50 \%$ and $44 \%$ in the FA-ref and BF-ref groups, respectively). Furthermore, ofatumumab was also active in those having a $17 \mathrm{p}$ deletion, with a response rate of $41 \%$ in the FA-ref group, although the response was lower at 14\% in the BF-ref group with $17 \mathrm{p}$ deletion. The median time to response was 1.8 months, and the median duration of response was 7.1 months in the FA-ref group and 5.6 months in the BF-ref group. Median progression-free survival and overall survival times were 5.7 and 13.7 months in the FA-ref group, respectively, and 5.9 and 15.4 months in the BF-ref group, respectively. The most common adverse events occurring during treatment were infections (67\%) and infusion-related reactions (seen in $64 \%$ of patients in the FA-ref group and $61 \%$ of patients in the BF-ref group), nearly all of which were Grade 1 or 2 . These reactions predominantly occurred during the first and second infusions, subsided during the course of treatment, and included cough (18\%), diarrhea (16\%), anemia (16\%), fatigue (15\%), fever (15\%), neutropenia $(15 \%)$, dyspnea $(13 \%)$, nausea $(11 \%)$, and rash $(10 \%)$. No human antihuman antibodies were detected in any of the evaluable patients. This study led to accelerated approval of ofatumumab in patients with CLL refractory to fludarabine and alemtuzumab by the US Food and Drug Administration in October 2009.

The final results for the primary endpoint of this study for 206 enrolled patients were reported at the 2010 American Society of Hematology annual meeting. The objective response rate by independent endpoint review committee evaluation was $51 \%$ for the FA-ref group and $44 \%$ for the BF-ref group. Two patients in the BF-ref group achieved a complete response. The median duration of response was 5.7 months in the FA-ref group and six months in the BF-ref group. Progression-free survival and overall survival were 5.5 months and 14.2 months for the FA-ref group, and 5.5 months and 17.4 months for the BF-ref group. ${ }^{14}$

Another single-arm Phase II study currently recruiting patients is investigating the effects of ofatumumab retreatment and maintenance in CLL patients who received the monoclonal antibody in a previous study (study number Hx-CD20-406). Patients will receive eight weekly infusions (300 mg, then seven $\times 2000 \mathrm{mg}$ doses), followed by $2000 \mathrm{mg}$ doses once a month for two years. The study is expected to enroll 25 patients, and primary outcome measures should be reported after January 2012 (clinicaltrials.gov study \#NCT00802737). 


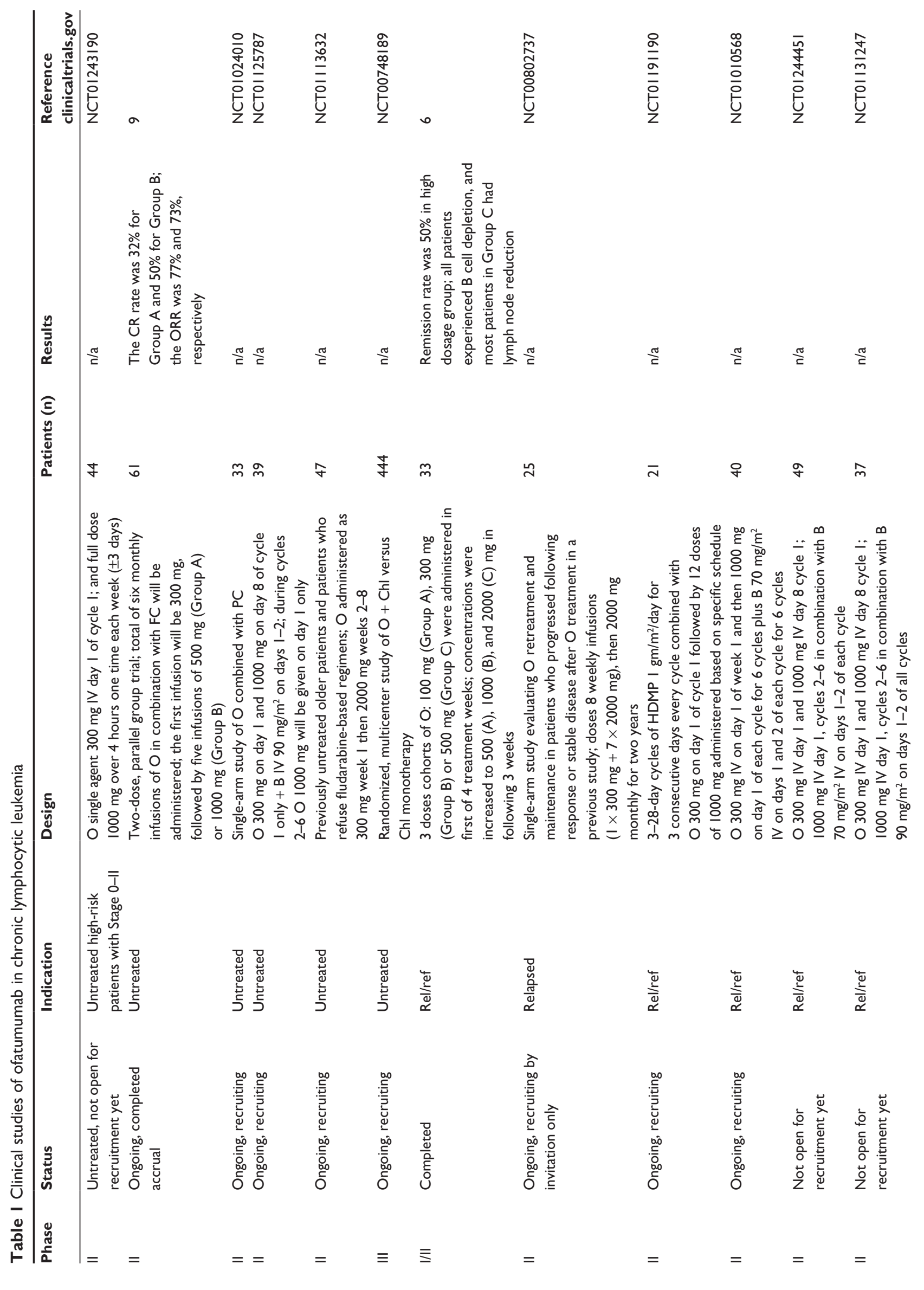




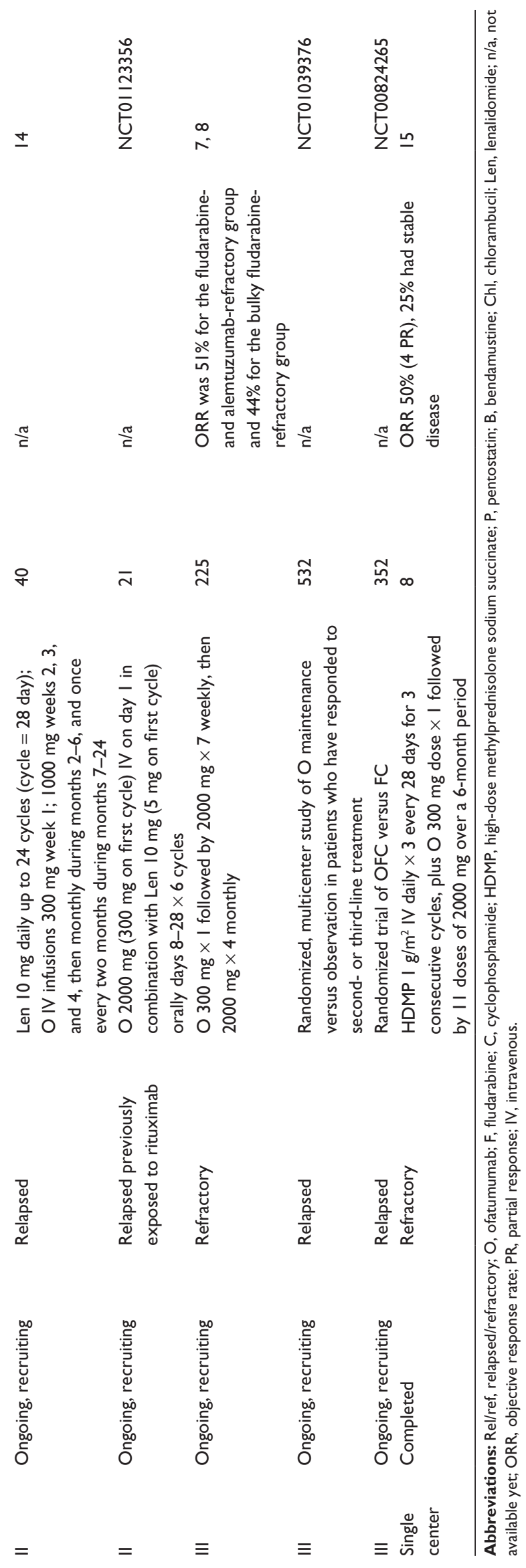

Following on from the single-agent results, studies evaluating ofatumumab combined with other chemotherapeutic agents have recently been undertaken. One of these studies was a two-dose parallel group, international, randomized, multicenter Phase II study looking at ofatumumab combined with fludarabine and cyclophosphamide (O-FC) in patients with previously untreated CLL. ${ }^{15} \mathrm{~A}$ total of 61 patients were randomized to receive ofatumumab $500 \mathrm{mg}$ (Group A) or $1000 \mathrm{mg}$ (Group B) on day 1, combined with fludarabine (25 mg/m² IV daily on days 1-3) and cyclophosphamide (250 mg/m² IV daily on days 1-3) every four weeks for a total of six courses. In both groups, the first dose of ofatumumab was $300 \mathrm{mg}$. The complete response rate was $32 \%$ for Group A and 50\% for Group B, and the objective response rate was $77 \%$ and $73 \%$, respectively. After a median follow-up duration of eight months, the median progressionfree survival has not been reached. This study demonstrates the activity of ofatumumab combined with fludarabine and cyclophosphamide in previously untreated patients with CLL, and although the objective response rate of O-FC was lower when compared with historical controls, the rate of complete response was higher when compared with patients having similar prognostic risk. Overall, the patients in the $\mathrm{O}-\mathrm{FC}$ trial were reportedly of high risk, which was primarily determined by an elevated $\beta_{2}$-microglobulin. Furthermore, a study evaluating 224 patients treated with fludarabine, cyclophosphamide, and rituximab in the frontline setting showed a complete response rate of $72 \%$ and an objective response rate of $95 \%$. However, this study had a lower number of patients with Rai Stage III-IV disease of $36 \%$ compared with $45 \%$ in the O-FC trial. ${ }^{16}$

Another study, recently reported at the 2010 American Society of Clinical Oncology meeting, showed a historic objective response rate of $92 \%$ and complete response rate of $66 \%$ for fludarabine, cyclophosphamide, and rituximab in previously untreated CLL patients. ${ }^{17}$ On the other hand, updated results of the German CLL Study Group for fludarabine and cyclophosphamide versus fludarabine, cyclophosphamide, and rituximab in the frontline setting revealed an objective response rate of $95.1 \%$ for the fludarabine, cyclophosphamide, and rituximab arm, but only a complete response rate of $44.1 \%{ }^{18}$ Another study evaluated bendamustine combined with rituximab in the frontline setting and showed an objective response rate of $90 \%$ with only a complete response rate of $32 \% .{ }^{19}$ Due to patient differences, it is hard to make comparisons using historical data. O-FC seems to be a promising and active regimen in the upfront setting, but Phase III data would likely be necessary to determine further its definite role. 
The initial results of a Phase II study evaluating the efficacy and tolerability of the combination of lenalidomide and ofatumumab in patients with relapsed CLL was recently presented at the 2010 American Society of Hematology meeting. ${ }^{20}$ In this trial, ofatumumab was administered IV weekly for four weeks (300 mg week 1, $1000 \mathrm{mg}$ week 2, and all subsequent doses), then monthly for months 2-6 and once every two months for months 7-24. Lenalidomide was given orally at a dose of $10 \mathrm{mg}$ daily, starting on day 9 and continued daily. The treatment duration was 24 months. Results for the first 16 of 40 planned patients who have been on study for at least three months were presented. Four patients $(25 \%)$ were refractory to fludarabine and all patients had received prior rituximab. Ten of the 16 evaluable patients achieved a response (two complete responses [13\%], eight partial responses [50\%]) for an objective response rate of $63 \%$. Four patients with stable disease were continuing on treatment. The most common Grade 3-4 treatment-related adverse events observed were neutropenia (eight patients, $50 \%$ ) and anemia (two patients, 13\%). Lenalidomideassociated tumor flare reaction was limited to Grade 1 in two patients (13\%). The authors concluded that the combination was therapeutically active in patients with relapsed CLL and was well tolerated.

The combination of high-dose methylprednisolone and ofatumumab was recently reported in patients with CLL not considered to be good candidates for chemotherapy due to comorbidities, poor performance status, profound cytopenia, or refractory status to fludarabine and/or alemtuzumab. ${ }^{21}$ Eight patients with progressive, symptomatic CLL were treated with high-dose methylprednisolone $1 \mathrm{~g} / \mathrm{m}^{2} \mathrm{IV}$ daily every 28 days for three consecutive cycles, and a single dose of ofatumumab $300 \mathrm{mg}$, followed by 11 doses of $2000 \mathrm{mg}$ over a six-month period. The median patient age was 69 years, and the median number of prior treatments was 4.5 , including four patients who had previously received high-dose methylprednisolone and rituximab and two patients who had undergone matched unrelated donor stem cell transplantation. All patients had been previously treated with rituximab, $75 \%$ had failed or were intolerant to fludarabine and/or alemtuzumab, and $75 \%$ had high-risk prognostic markers, including unfavorable cytogenetics, unmutated $\operatorname{IgV}_{\mathrm{H}}$ region genes, or high expression levels of zeta-associated protein. Most of the patients had bulky disease and splenomegaly. All patients completed the planned therapy with no major side effects or toxicities. There was no evidence of marrow suppression, and even patients with pancytopenia improved their peripheral blood counts with this salvage regimen.
The objective response rate was 50\% (four partial remissions), $25 \%$ of patients had stable disease, and the remainder showed progressive disease. These data suggest that the combination of high-dose methylprednisolone and ofatumumab is a safe and effective salvage regimen for high-risk CLL patients who otherwise would not be candidates for additional treatment. Further clinical studies of this combination are warranted.

\section{Conclusion}

Ofatumumab has significant antileukemic activity, and offers another effective agent with which to improve the outcome of patients with CLL/SLL. Further studies will clarify the optimal dose and time to use this drug. CLL refractory to fludarabine is a high-risk disease that has very few treatment options. In this regard, ofatumumab seems to be an effective agent. However, in the previously untreated CLL setting, we do not believe any of the current studies can justify using this therapy over one of the other more established and costeffective regimens, such as fludarabine, cyclophosphamide, and rituximab. Ongoing clinical trials will determine the role of ofatumumab in CLL in both the upfront and the relapsed setting. Furthermore, randomized Phase III clinical trials will be required to determine if ofatumumab is a clinical advance over rituximab.

\section{Disclosure}

The authors report no conflicts of interest in this work.

\section{References}

1. National Cancer Institute. SEER Stat Fact Sheets: Chronic lymphocytic leukemia. Available from: http://seer.cancer.gov/statfacts/html/clyl.html. Accessed May 1, 2010.

2. Keating MJ, O'Brien S, Lerner S, et al. Long-term follow-up of patients with chronic lymphocytic leukemia (CLL) receiving fludarabine regimens as initial therapy. Blood. 1998;92(4):1165-1171.

3. Lipshutz MD, Mir R, Rai KR, Sawitsky A. Bone marrow biopsy and clinical staging in chronic lymphocytic leukemia. Cancer. 1980;46(6): 1422-1427.

4. Wierda WG, O'Brien S, Wang X, et al. Characteristics associated with important clinical end points in patients with chronic lymphocytic leukemia at initial treatment. J Clin Oncol. 2009;27(10):1637-1643.

5. Dohner H, Stilgenbauer S, Benner A, et al. Genomic aberrations and survival in chronic lymphocytic leukemia. N Engl J Med. 2000;343(26): 1910-1916.

6. Teeling JL, French RR, Cragg MS, et al. Characterization of new human CD20 monoclonal antibodies with potent cytolytic activity against nonHodgkin lymphomas. Blood. 2004;104(6):1793-1800.

7. Cragg MS, Walshe CA, Ivanov AO, Glennie MJ. The biology of CD20 and its potential as a target for mAb therapy. Curr Dir Autoimmun. 2005;8:140-174.

8. Polyak MJ, Deans JP. Alanine-170 and proline-172 are critical determinants for extracellular CD20 epitopes; heterogeneity in the fine specificity of CD20 monoclonal antibodies is defined by additional requirements imposed by both amino acid sequence and quaternary structure. Blood. 2002;99(9):3256-3262. 
9. Barth M, Hernandez-Ilizaliturri FJ, Mavis C, et al. Ofatumumab, a fully human monoclonal antibody targeting CD20, demonstrates activity against and potentiates the anti-tumor activity of chemotherapy agents in rituximab-sensitive cell lines (RSCL), rituximab-resistant cell lines (RRCL), lymphoma xenografts, and primary tumor cells derived from patients with B-cell non-Hodgkin lymphoma (NHL). Blood. 2010; 116(21):3917.

10. Teeling JL, Mackus WJ, Wiegman LJ, et al. The biological activity of human CD20 monoclonal antibodies is linked to unique epitopes on CD20. J Immunol. 2006;177(1):362-371.

11. Bleeker WK, Munk ME, Mackus WJ, et al. Estimation of dose requirements for sustained in vivo activity of a therapeutic human anti-CD20 antibody. Br J Haematol. 2008;140(3):303-312.

12. Coiffier B, Lepretre S, Pedersen LM, et al. Safety and efficacy of ofatumumab, a fully human monoclonal anti-CD20 antibody, in patients with relapsed or refractory B-cell chronic lymphocytic leukemia: A phase 1-2 study. Blood. 2008;111(3):1094-1100.

13. Wierda WG, Kipps TJ, Mayer J, et al. Ofatumumab as single-agent CD20 immunotherapy in fludarabine-refractory chronic lymphocytic leukemia. J Clin Oncol. 2010;28(10):1749-1755.

14. Wierda WG, Kipps TJ, Mayer J, et al. Final analysis from the international trial of single-agent ofatumumab in patients with fludarabine-refractory chronic lymphocytic leukemia. Blood. 2010; 116(21):921.

15. Wierda WG, Kipps TJ, Durig J, et al. Ofatumumab combined with fludarabine and cyclophosphamide (O-FC) shows high activity in patients with previously untreated chronic lymphocytic leukemia (CLL): Results from a randomized, multicenter, international, two-dose, parallel group, Phase II trial. Blood. 2009;114(22):207.
16. Tam CS, O'Brien S, Wierda W, et al. Long-term results of the fludarabine, cyclophosphamide, and rituximab regimen as initial therapy of chronic lymphocytic leukemia. Blood. 2008;112(4):97-80.

17. Parikh SA, Wierda WG, Badoux X, et al. Comparison of fludarabine $(F)$ plus cyclophosphamide (C) versus FC plus rituximab (R) in previously untreated Rai Stage III/IV chronic lymphocytic leukemia (CLL). J Clin Oncol. 2010;28 Suppl 15:6519.

18. Hallek M, Fingerle-Rowson G, Fink A-M, et al. First-line treatment with fludarabine (F), cyclophosphamide (C), and rituximab (R) (FCR) improves overall survival (OS) in previously untreated patients (pts) with advanced chronic lymphocytic leukemia (CLL): Results of a randomized Phase III trial on behalf of an international group of investigators and the German CLL Study Group. Blood. 2009; 114(22):535.

19. Fischer K, Cramer P, Stilgenbauer S, et al. Bendamustine combined with rituximab (BR) in first-line therapy of advanced CLL: A multicenter Phase II trial of the German CLL Study Group (GCLLSG). Blood. 2009;114(22):205

20. Badoux X, O'Brien S, Wierda WG, et al. Combination of ofatumumab and lenalidomide in patients with relapsed chronic lymphocytic leukemia: Initial results of a Phase II trial. Blood. 2010;116(21):2464.

21. Castro JE, Barajas-Gamboa JS, Melo-Cardenas J, et al. Ofatumumab and high-dose methylprednisolone is an effective salvage treatment for heavily pretreated, unfit or refractory patients with chronic lymphocytic leukemia: Single institution experience. Blood. 2010;116(21):4638.

\section{Publish your work in this journal}

The Journal of Blood Medicine is an international, peer-reviewed, open access, online journal publishing laboratory, experimental and clinical aspects of all topics pertaining to blood based medicine including but not limited to: Transfusion Medicine; Blood collection, Donor issues, Transmittable diseases, and Blood banking logistics; Immunohematology; Artificial and alternative

\section{Dovepress}

blood based therapeutics; Hematology; Biotechnology/nanotechnology of blood related medicine; Legal aspects of blood medicine; Historical perspectives. The manuscript management system is completely online and includes a very quick and fair peer-review system. Visit http://www.dovepress.com/ testimonials.php to read real quotes from published authors. 